\title{
Learning-related activation in the auditory system of the rat produced by long-term habituation: a 2-deoxyglucose study
}

\author{
F. Gonzalez-Lima ${ }^{1}$, T. Finkenstädt ${ }^{2}$ and J.-P. Ewert ${ }^{2}$ \\ ${ }^{1}$ Department of Anatomy, College of Medicine, Texas A\&M University, College Station, TX 77843 (U.S.A.) and ${ }^{2}$ Department of \\ Neuroethology, University of Kassel, Kassel (F.R.G.)
}

(Accepted 15 November 1988)

Key words: Auditory system; Long-term habituation; Acoustic startle response; Learning; 2-Deoxyglucose

\begin{abstract}
Autoradiography with $\left[{ }^{14} \mathrm{C}\right] 2$-deoxyglucose (2-DG) was used to examine the functional activity of the rat auditory system during long- and short-term habituation of the acoustic startle reflex. The data showed that presentation of the acoustic stimulus to long-term habituated rats resulted in a learning-related metabolic enhancement that was significantly greater than the response evoked by the same acoustic stimulus in the inexperienced rats. This enhancement was localized to brainstem and midbrain auditory nuclei and no significant changes occurred at thalamocortical levels of the auditory pathway. The largest difference in 2-DG uptake between long- and short-term habituated rats was in the lateral superior olivary nucleus (LSO). The LSO activation suggests that olivocochlear efferents may operate in a central feedback control of peripheral auditory input during long-term habituation. Findings of enhanced metabolism from the cochlear nuclei to the central nucleus of the inferior colliculus indicated that active processes of neuronal plasticity take place in the lower auditory system during long-term habituation. The results provide the first demonstration of how a nonassociative learning experience such as long-term habituation modifies the metabolic activity of the auditory system. The findings support the conclusion that auditory responses of behaving animals to acoustic stimuli are dependent not only on the physical parameters of a stimulus, but also on its learned behavioral significance.
\end{abstract}

\section{INTRODUCTION}

Autoradiography with $\left[{ }^{14} \mathrm{C}\right] 2$-deoxyglucose (2DG) has allowed the visualization of neural patterns of functional activity related to behavior in various species of animals ${ }^{4-10,12,23,26}$. The 2-DG technique uses trace amounts of a labeled glucose analog (2-DG) that becomes trapped inside brain cells ${ }^{23}$. Since glucose is the primary energy source for brain cells, the mapping of 2-DG uptake visualizes regions with function-dependent changes in activity. We have recently used a 2-DG approach in a series of behavioral studies for mapping brain structures influenced by arousal, sensitization and Pavlovian associative learning ${ }^{4-9}$. These studies showed that learning-related changes in 2-DG uptake can be differentiated from changes related to arousal and sensitization in the auditory system. Our 2-DG findings suggested that auditory learning represents a distributed property of neuronal maps which involves different levels of the auditory pathway ${ }^{6,8}$. The question remained of how a simple form of nonassociative learning, such as habituation, would modify neural metabolic activity in the auditory system.

Regarded as one of the simplest and most universal types of learning, habituation may be defined as the decrease of a response as a consequence of repeated presentation of a stimulus ${ }^{13,25}$. A distinction of two independent behavioral processes with different underlying neural mechanisms has been made between short- (within session) and long-term (across sessions) habituation ${ }^{2,16-18}$. Short-term habituation is a transient process which recovers spontaneously following stimulus removal ${ }^{2,3,15}$. Long-term habituation is a more permanent behav-

Correspondence: F. Gonzalez-Lima, Department of Anatomy, College of Medicine, Texas A\&M University, College Station, TX 77843 , U.S.A. 
ioral decrement occurring after daily sessions of stimulation $^{16-18}$. A previous 2-DG study of photic habituation showed increases in glucose metabolism in visual structures in a comparison of rats injected with 2-DG at the beginning or at the end of a single habituation session ${ }^{26}$. The 2-DG method can also be used in an analogous manner to determine whether auditory structures are involved in long-term habituation. Two groups of intact rats may be exposed to identical acoustic stimulation after injection of 2DG. One group of rats may be habituated to the stimulus for several days, while the other may have no experience with the stimulus. Differences in 2-DG uptake patterns evoked in the auditory system in response to the same acoustic stimulus would then be attributed to plasticity changes produced by long-term habituation.

Great attention has been given to the acoustic startle response in rats as a model for investigating the neural basis of habituation ${ }^{2,3 \cdot 16-19}$. A full-body startle reflex can be elicited in rats by a loud noise. With repeated presentations of the noise, the startle response decreases progressively. The acoustic startle response shows both short- and long-term decrements to repeated stimuli. It is well established that neural mechanisms of short-term habituation of the acoustic startle reflex are intrinsic to the stimulus-response reflex circuit in the lower brainstem ${ }^{2,3}$. This primary circuit consists of: auditory nerve, ventral cochlear nucleus, nuclei of the lateral lemniscus, nucleus reticularis pontis caudalis, spinal interneuron, ventral horn motoneuron, and muscles $^{3}$. In the case of long-term habituation, however, lesion studies imply that its circuitry may involve auditory structures extrinsic to the primary acoustic startle reflex circuit ${ }^{16-19}$. In an attempt to further explore the question of auditory system plasticity in long-term habituation ${ }^{10}$, the 2-DG method was used in the present study to map the functional activity of the intact rat brain during longas compared to short-term habituation of the acoustic startle reflex.

\section{MATERIALS AND METHODS}

\section{Subjects}

Thirty-three male albino rats of $100 \mathrm{~g}$ body weight were used. First, 20 rats were divided into several groups receiving long-term and short-term habituation in a series of preliminary behavioral experiments. Second, another 13 rats were used for the 2-DG experiment following the most compatible schedule for startle habituation determined from the preliminary behavioral experiments as explained below. All subjects were handled in the same way and they were housed two per cage in the same room under constant environmental control and freely available water and food.

\section{Apparatus}

A startle cage of $18 \times 15 \times 15 \mathrm{~cm}$ within a firm steel frame similar to the apparatus described by Cassella and Davis ${ }^{1}$, was fitted with a myograph connected to a transducer coupler (Narco models F-2000 and 7173). Movement of the cage detected by the myograph and transducer was amplified (Narco amplifier $7070,30 \mathrm{~Hz}$ filter) and recorded by pen deflection in a polygraph (Narco physiograph CPM). The amplitude of the full-body startle response as detected by the pen recording was proportional to cage movement. The acoustic startle response was measured after $100 \mathrm{~dB}, 1 \mathrm{~s}$ bursts of white noise superimposed on a $55 \mathrm{~dB}$ background white noise. Sound intensity was measured at the center of the cage with a sound level meter (Simpson 884-52A). The sound was produced by generators (Narco SI-10), amplified by an audio system (Technics) connected to two speakers, and triggered by a computer (IBM PC).

\section{Treatments}

Fully awake and unrestrained rats received an i.p. injection of $18 \mu \mathrm{Ci} / 100 \mathrm{~g}$ body weight of $\left[{ }^{14} \mathrm{C}\right] 2$ deoxy-D-glucose (spec. act. of $300-360 \mathrm{mCi} / \mathrm{mM}$ ) in $0.2 \mathrm{ml}$ sterile $0.9 \%$ saline. They were placed in the startle cage and after the $45 \mathrm{~min}$ exposure to acoustic stimulation (or no stimulation control) under 2-DG, each rat was decapitated and the brain quickly removed and frozen.

Each stimulation session lasted $45 \mathrm{~min}$ (to be compatible with the 2-DG method) and consisted of 270 noise bursts. One air-puff was also presented as the last stimulus before removal of the rat for decapitation. The large startle response evoked by the air-puff served to verify that in each rat the decrements in acoustic startle responding were due 
to stimulus-specific habituation. This ruled out the possibility of fatigue and nonspecific motor effects as the explanation for the decrement in startle responding to repeated acoustic stimulation. Acoustic stimuli were presented at irregular intervals of $10 \mathrm{~s}$ on average (4-16 $\mathrm{s}$ range). Irregular intervals were used to rule out the possibility of temporal conditioning. This schedule was chosen from preliminary experiments because it allowed the maximum number of stimulus presentations after 2-DG injection. A relatively large number of presentations yielded optimal effects in the amount of 2-DG uptake. Rats were randomly assigned to one of 3 groups.

Group 1. Rats $(n=5)$ were long-term habituated for 5 consecutive days ( 2 sessions/day separated by 1 h) and were injected with 2-DG immediately before their 9th stimulation session. This group was used to determine the effects of long-term habituation on the uptake of 2-DG evoked by the acoustic stimulus in the auditory system.

Group 2. Rats $(n=5)$ were not long-term habituated and had no experience with the acoustic stimulus; but they were otherwise handled like rats of Group 1. Then they were injected with 2-DG prior to their first and only session of short-term habituation with the acoustic stimulus. This group was used to compare the effects of short-term habituation to the acoustic stimulus with the effects produced by long-term habituation. Since Groups 1 and 2 rats received identical acoustic stimulation during the 2-DG exposure session, differences in startle responses and auditory patterns of 2-DG uptake would then derive from learning-related changes.

Group 3. Rats $(n=3)$ were injected with 2-DG and served as unstimulated controls. Two kinds of control rats were used to rule out the possibility of nonspecific pretraining effects on the auditory system. One rat was pretrained with long-term habituation (as in Group 1) while the others were not pretrained (as in Group 2). Since none of the control rats were subjected to the acoustic stimulus after 2-DG injection, they all showed essentially the same uptake of 2-DG in the auditory system (mean \pm S.E.M. in Table II). This control procedure served to ascertain that 2-DG increases in the auditory system were stimulus-dependent.

\section{Autoradiography}

The brains were processed for 2-DG autoradiography as previously described ${ }^{5-9}$. Briefly, the whole frozen brain was cut transversely in serial sections of $40 \mu \mathrm{m}$ so that the auditory pathway could be analyzed at all levels. Sections were picked up with slides and dried on a hotplate at $60^{\circ} \mathrm{C}$. After the slides were glued to cardboard, Kodak NMB film was placed above the brain sections, and stored inside Kodak X-O-Matic cassettes for 2 weeks. Films were developed in Kodak D-19 for 2 min, rinsed 1 $\mathrm{min}$ in $2 \%$ acetic acid, fixed $10 \mathrm{~min}$, washed $15 \mathrm{~min}$, and dried. In each film, plastic micro-scale standards of known ${ }^{14} \mathrm{C}$ concentrations $(40-1070 \mathrm{nCi} / \mathrm{g}$ tissue, Amersham) were developed along with the sections. The standards were used to calculate ${ }^{14} \mathrm{C}$ concentration values in the tissue. After obtaining the autoradiographic images, the tissue sections were stained with Cresyl violet for histological correlation between the functional and morphological images.

\section{Quantitative image analysis}

Incorporation of 2-DG was quantified with the image-processing system Quantimet 920 (Cambridge Instruments) as explained previously ${ }^{4}$. Briefly, images from each brain were digitized and calibrated with the ${ }^{14} \mathrm{C}$ standards to determine isotope incorporation. The system provided: optical density, calculated isotope content, ratio between the labeling of each measured structure and the white matter of the optic tract (used as reference), and relative optical density index between the structure and the entire section. It was determined that there were no significant differences in labeling in the optic tract white matter between any of the groups (Groups 1 vs $2, P=0.38$; Groups 1 vs $3, P=0.67$; and Groups 2 vs $3, P=0.38$; Kolmogorov-Smirnoff-Omnibus test). Therefore, the values in Table II are expressed as gray/white matter ratios of 2-DG uptake of a given structure divided by that of the optic tract for that brain. This analytical approach is based on relative differences in tissue labeling with no intention of estimating rates of glucose utilization, but rather measuring relative changes in functional activity in the auditory system ${ }^{23}$. Further transformation of 2-DG autoradiographic data to estimate absolute rates of glucose utilization is a matter of controversy, and it is unnecessary for our objective 
of obtaining a relative index of differences in brain metabolic activity between short- and long-term habituated rats $^{23}$. No subjective judgment entered into the determination of significant 2-DG labeling. The structures were delineated morphologically with a graphics tablet and the 2-DG values and statistics were automatically printed by the imaging system. In every structure, 2-DG values were averaged for at least 3 consecutive sections in each brain. The mean 2-DG uptake ratios contributed by every rat in a group were averaged to give the final statistics. Mann-Whitney $U$-tests for ratios were used to assess statistical differences between groups.

\section{RESULTS}

\section{Behavioral results}

Table I shows that the average startle amplitude for the short-term habituated rats was $22.42 \pm 3.17$. This corresponded to a $25.26 \%$ reduction score relative to the initial startle response of $30.00 \pm 5.99$. In the case of long-term habituated rats, their average startle amplitude was $14.59 \pm 1.97$ in the 2-DG session. This corresponded to a $51.36 \%$ reduction score relative to the initial startle response. Therefore, the long-term rats showed a response decrement twice as large as the decrement found in the short-term rats (significant difference, $t$ $=5.03, P<0.01$ ). Furthermore, the control trials with air-puffs showed that the group difference was stimulus-specific to the acoustic stimulus. The mean startle amplitude produced by the terminal air-puff stimulus was $33.30 \pm 4.04$ as compared to the initial mean amplitude of $30.00 \pm 5.99$ produced by the first acoustic stimulus (no significant differences). These data served as behavioral evidence of the learning effect of long-term habituation. That is, rats in Groups 1 and 2 received identical acoustic stimulation during the 2-DG session, but long-term habituated rats showed a much greater suppression of startle responding due to their previous daily experiences with acoustic stimulus.

\section{Autoradiographic results}

Table II gives the magnitude of the metabolic changes in each auditory structure for Groups 1-3. The auditory system of the long-term group, when compared to the short-term group, revealed clearly enhanced regional metabolic patterns in response to identical acoustic stimulation during the 2-DG session.

\section{Cochlear nuclei}

Fig. 1 shows the analysis of representative autoradiographs chosen from comparable anteroposterior locations of the dorsal (DCN) and ventral (VCN) cochlear nuclei. Uptake of 2-DG was always greater in the DCN than in the VCN in Groups 1-3. The long-term habituated rats showed the largest activity followed by the short-term and the unstimulated control groups. This progressive sequence of labeling can be observed in Fig. 1B which shows binary-coded autoradiographic images converted to gray scales, black meaning strongest 2-DG uptake. The mean differences were significant $(P<0.05)$ between the long- and short-term groups and between each of these two groups and the controls (Table II). Therefore, presentation of the acoustic stimulus to long-term habituated rats resulted in a learning-related metabolic enhancement that was significantly greater than the response evoked by the same acoustic stimulus in the inexperienced rats.

\section{Superior olivary-trapezoid body complex}

The lateral superior olivary nucleus (LSO) showed the largest increase in long-term rats when compared to short-term rats (Table II). The percent increase in the LSO between long-term and control groups $(30 \%)$ was over 4 times greater than the increase found between the short-term and control groups $(7 \%)$. This provided evidence of a powerful metabolic effect on the LSO produced by the acoustic stimulus as a result of long-term habituation. This effect is illustrated in Fig. 2 which shows autoradiographs at the level of the lateral (LSO) and medial (MSO) nuclei of the superior olive, and the medially adjacent nucleus of the trapezoid body (TB). All of these nuclei showed significantly greater activity in the long-term group, followed by the short-term and the control groups. But the relative increase between long- and short-term groups is greatest in the LSO (22\%) as compared to all the other auditory structures (Table II). This suggests that activity changes in the LSO may play a particularly significant role in long-term habituation of the acoustic startle response. 


\section{TABLE I}

Mean startle amplitudes of long-term habituated $(n=5)$ and short-term habituated $(n=5)$ groups of rats

The values are acoustic startle amplitudes over each block of trials following injection of 2-DG. Mean values were obtained by averaging responses contributed by every rat in a group. Percentages of startle amplitudes (relative to the initial acoustic startle response) are also presented for each block of trials and for the average total response in each group.

\begin{tabular}{|c|c|c|c|c|c|}
\hline \multirow[t]{2}{*}{ Time (min) } & \multirow[t]{2}{*}{ Trials } & \multicolumn{2}{|c|}{ Startle amplitude (mean \pm S.E.M.) } & \multicolumn{2}{|c|}{ Percent of initial startle } \\
\hline & & Long & Short & Long & Short \\
\hline 0 & 1 & $20.70 \pm 3.53$ & $30.00 \pm 5.99$ & 69.00 & 100.00 \\
\hline 5 & $2-30$ & $12.73 \pm 0.93$ & $27.61 \pm 5.61$ & 42.43 & 92.03 \\
\hline 10 & $31-60$ & $13.00 \pm 1.24$ & $24.32 \pm 8.12$ & 43.33 & 81.05 \\
\hline 15 & $61-90$ & $13.33 \pm 1.40$ & $24.81 \pm 6.30$ & 44.43 & 82.69 \\
\hline 20 & $91-120$ & $13.31 \pm 1.17$ & $19.00 \pm 2.69$ & 44.38 & 63.33 \\
\hline 25 & $121-150$ & $14.54 \pm 1.59$ & $19.44 \pm 3.20$ & 48.48 & 64.81 \\
\hline 30 & $151-180$ & $16.12 \pm 1.60$ & $19.39 \pm 2.67$ & 53.73 & 64.62 \\
\hline 35 & $181-210$ & $18.43 \pm 2.90$ & $19.52 \pm 3.06$ & 61.44 & 65.07 \\
\hline 40 & $211-240$ & $16.47 \pm 2.46$ & $24.93 \pm 7.33$ & 54.91 & 83.24 \\
\hline 45 & $241-270$ & $13.17 \pm 1.51$ & $22.81 \pm 5.87$ & 43.89 & 76.03 \\
\hline \multicolumn{2}{|c|}{ Average total trials* } & $14.59 \pm 1.97$ & $22.42 \pm 3.17$ & 48.64 & 74.74 \\
\hline
\end{tabular}

* Significant group difference at $P<0.01, t$-test.

\section{Nuclei of the lateral lemniscus}

Presentation of the acoustic stimulus during shortterm habituation leads to very little increase $(4 \%$, not significant) in 2-DG uptake in the nuclei of the lateral lemniscus (LL) when compared to the unstimulated controls. This was the smallest increase produced by short-term acoustic stimulation in the auditory system (Table II). In addition, no signifi-

\section{TABLE II}

Relative 2-DG uptake of auditory structures in a comparison of long-term habituated $(n=5)$, short-term habituated $(n=5)$ and control $(n=3)$ groups of rats

The values are gray/white matter ratios of 2-DG uptake of a given structure divided by that of the optic tract for that brain. Mean values were obtained by averaging the ratios contributed by every rat in a group. Percent differences between the groups were calculated with the formula: $100 \times$ (Long-Short)/Short, $100 \times$ (Long-Control)/Control, and 100 $\times$ (Short-Control)/Control. Mann-Whitney $U$-tests for ratios were used to assess differences.

\begin{tabular}{|c|c|c|c|c|c|c|}
\hline \multirow[t]{2}{*}{ Structure } & \multicolumn{3}{|c|}{ Relative 2-DG uptake (mean \pm S.E.M.) } & \multicolumn{3}{|c|}{ Differences between groups (\%) } \\
\hline & Long & Short & Control & Long-Short & Long-Control & Short-Control \\
\hline Dorsal cochlear n. (DCN) & $2.16 \pm 0.08$ & $1.90 \pm 0.07$ & $1.70 \pm 0.06$ & $13.70^{*}$ & $27.11^{*}$ & $11.80^{*}$ \\
\hline Ventral cochlear n. (VCN) & $1.87 \pm 0.08$ & $1.59 \pm 0.06$ & $1.41 \pm 0.01$ & $17.28^{*}$ & $31.95^{*}$ & $12.51^{*}$ \\
\hline Trapezoid body n. (TB) & $1.71 \pm 0.07$ & $1.47 \pm 0.07$ & $1.39 \pm 0.07$ & $16.19^{*}$ & $23.02^{*}$ & 5.87 \\
\hline $\begin{array}{l}\text { Lateral superior olivary } n \text {. } \\
\text { (LSO) }\end{array}$ & $2.19 \pm 0.12$ & $1.80 \pm 0.05$ & $1.68 \pm 0.09$ & $21.80^{* *}$ & $30.15^{*}$ & 6.86 \\
\hline $\begin{array}{l}\text { Medial superior olivary } n \text {. } \\
\text { (MSO) }\end{array}$ & $2.03 \pm 0.09$ & $1.78 \pm 0.07$ & $1.62 \pm 0.10$ & $14.10^{*}$ & $25.49^{*}$ & 9.99 \\
\hline $\begin{array}{l}\text { Lateral lemniscus } n \text {. (LL) } \\
\text { Inferior colliculus, central n }\end{array}$ & $1.80 \pm 0.08$ & $1.61 \pm 0.04$ & $1.54 \pm 0.06$ & $12.07^{*}$ & $16.77^{*}$ & 4.19 \\
\hline $\begin{array}{l}\text { Inferior colliculus, external n. } \\
\text { (ICE) } \\
\text { Medial geniculate n. (MG) } \\
\text { Auditory cortex (AC) }\end{array}$ & $\begin{array}{l}1.66 \pm 0.06 \\
1.76 \pm 0.06 \\
1.78 \pm 0.04\end{array}$ & $\begin{array}{l}1.55 \pm 0.04 \\
1.80 \pm 0.05 \\
1.80 \pm 0.04\end{array}$ & $\begin{array}{l}1.46 \pm 0.02 \\
1.64 \pm 0.03 \\
1.70 \pm 0.05\end{array}$ & $\begin{array}{r}6.96 \\
-\quad 2.05 \\
-0.87\end{array}$ & $\begin{array}{c}13.49^{*} \\
7.40 \\
4.92\end{array}$ & $\begin{array}{l}6.10 \\
9.65^{*} \\
5.84^{*}\end{array}$ \\
\hline
\end{tabular}

\footnotetext{
* Significant difference at $P<0.05$.

** Significant difference at $P<0.01$.
} 
cant differences were observed in the pontine reticular formation ( $\mathrm{pr}$ ) between the short-term and control groups (Fig. 3). The pontine reticular formation is assumed to receive the outflow of the $\mathrm{LL}$ nuclei and project to the spinal cord as part of the output limb of the primary acoustic startle reflex. Table II shows that among the auditory nuclei of the brainstem, only the cochlear nuclear complex
LONG
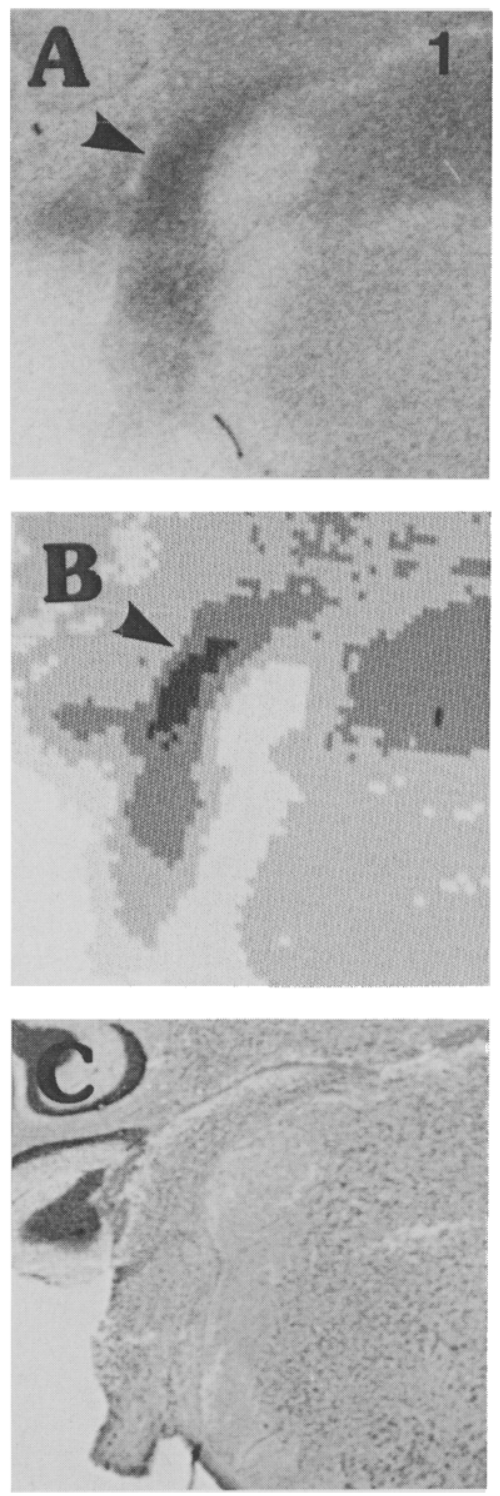

SHORT
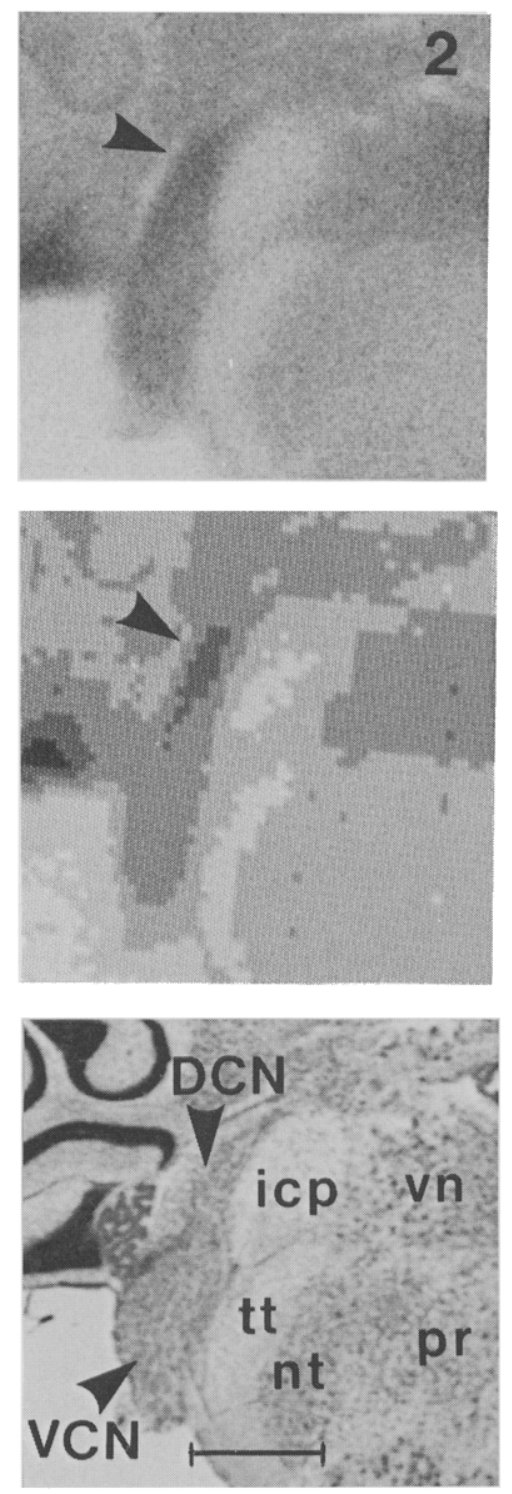

CONTROL
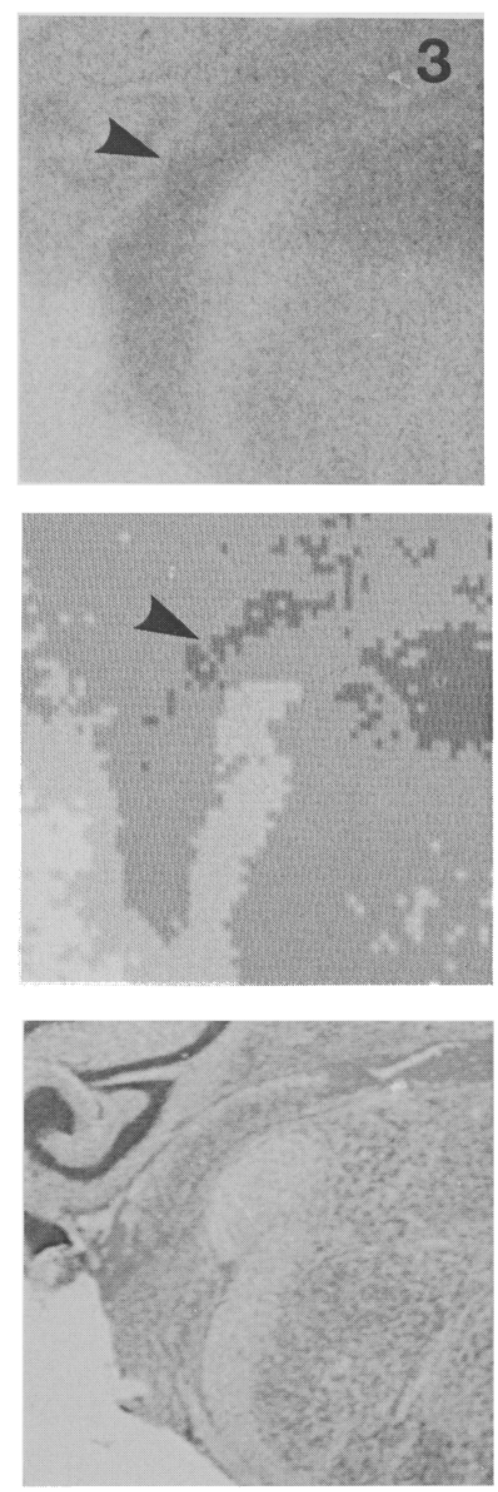

Fig. 1. Incorporation of 2-DG in the cochlear nuclei of rats from Group 1 (column 1, long), Group 2 (column 2, short) and Group 3 (column 3, control). Row A shows 2-DG autoradiographs at comparable anteroposterior levels from rats subjected to long-term habituation $\left(A_{1}\right)$, short-term habituation $\left(A_{2}\right)$ and untreated controls $\left(A_{3}\right)$. Row $B$ are computer-generated densitometric plots of the autoradiographs converted to gray scales, black meaning strongest 2-DG uptake. The arrowheads in $A$ and $B$ point to the same region of the DCN represented in the two different displays. Row $\mathrm{C}$ are the tissue sections used to produce the autoradiographs. All sections were stained with a Nissl stain after development of the autoradiographic images in order to match precisely tissue morphology with 2-DG labeling. VCN, ventral cochlear $n$.; DCN, dorsal cochlear $n$.; icp, inferior cerebellar peduncle; vn, vestibular $\mathrm{n}$.; pr, pontine reticular formation; $\mathrm{tt}$, trigeminal spinal tract; $\mathrm{nt}$, nucleus of $\mathrm{tt}$. $\mathrm{Bar}=1 \mathrm{~mm}$. 
(DCN, VCN) showed significant increases in the comparison between short-term and control groups. The other brainstem auditory nuclei (LL, LSO, MSO, TB) showed smaller changes that failed to reach statistical significance. This is particularly relevant in view of the involvement of LL nuclei in the primary circuit for the acoustic startle reflex. Therefore, auditory plasticity changes related to short-term habituation may be more likely localized to the cochlear than the LL nuclear components of the acoustic startle circuit.

In contrast, presentation of the acoustic stimulus to long-term habituated rats resulted in a significant increase $(17 \%)$ in LL labeling when compared to controls. This increase in LL was over 4 times greater than the corresponding percent difference

\section{LONG}
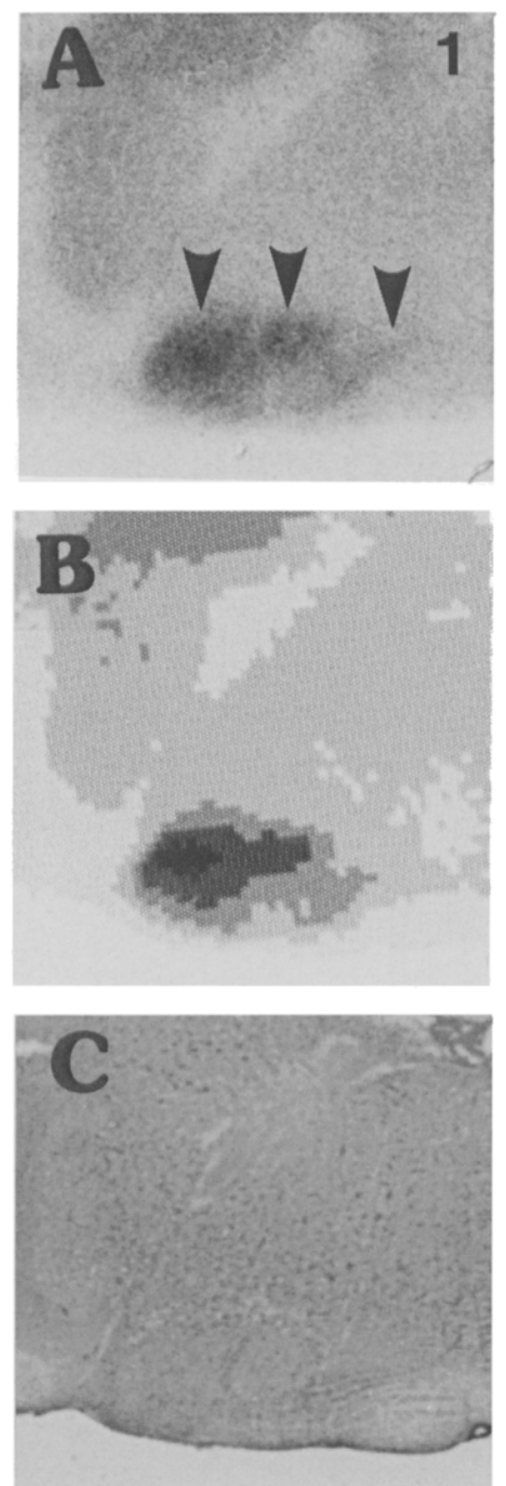

SHORT
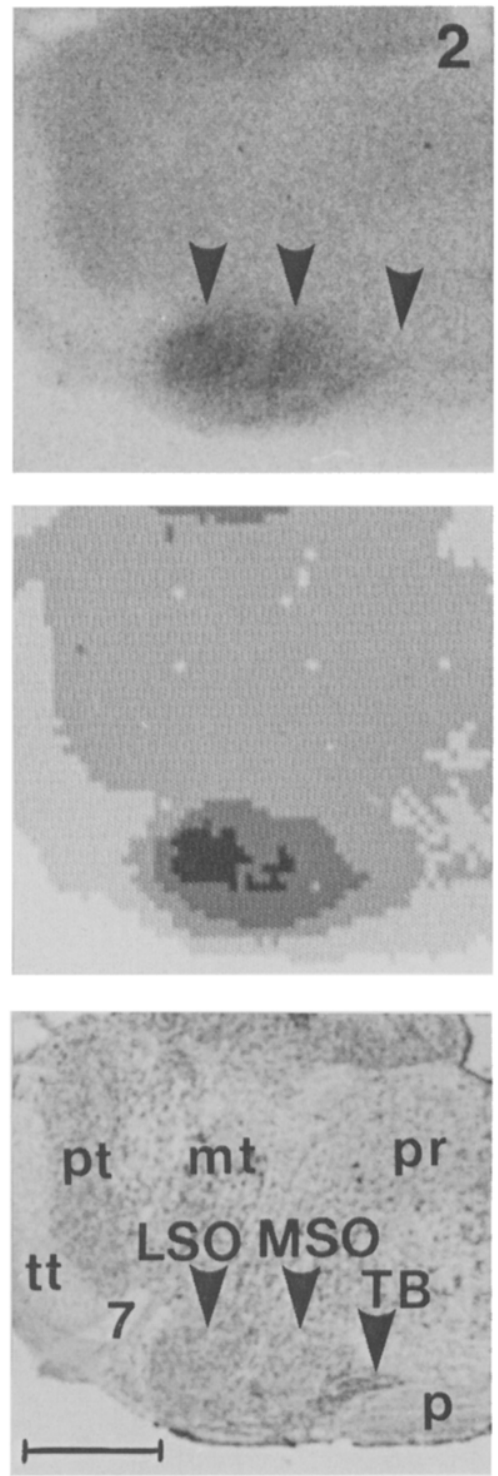

CONTROL
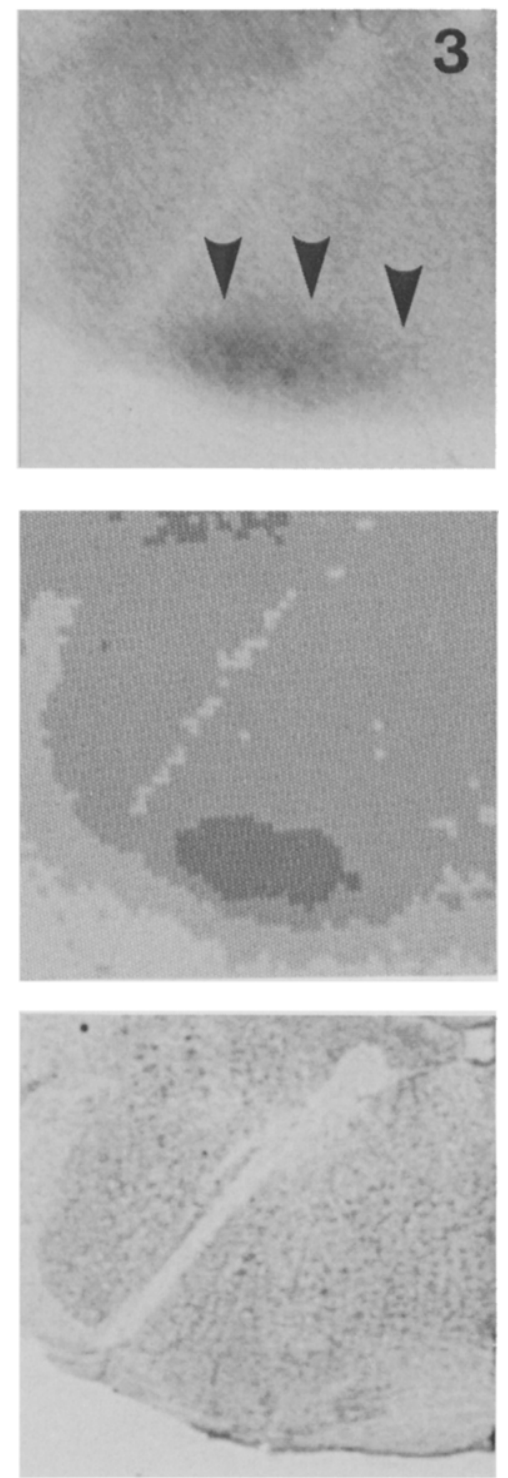

Fig. 2. Incorporation of 2-DG in the superior olivary-trapezoid body complex of rats from Groups 1-3. The organization of the figure is the same as in Fig. 1. The three arrowheads in each autoradiograph of row A point to the LSO, MSO, and TB from lateral to medial, respectively. The region of maximal labeling corresponded to LSO in the long-term habituated rat (LONG). LSO, lateral superior olivary n.; MSO, medial superior olivary n.; TB, trapezoid body $n$.; $p$, pyramidal tract; 7 , cranial nerve 7; tt, trigeminal sensory root; pt, principal sensory trigeminal n.; mt, motor trigeminal $\mathrm{n}$; pr, pontine reticular formation. Bar $=1 \mathrm{~mm}$. 
(4\%) between the short-term and control groups. These effects were more evident in the ventral (VLL) than in the dorsal (DLL) nuclei of the LL, as illustrated in Fig. 3. Therefore, 2-DG changes related to long-term habituation were localized in auditory brainstem nuclei intrinsic (VCN, LL) as well as extrinsic (LSO, MSO, TB, DCN) to the primary acoustic startle circuit.

\section{Inferior colliculus}

The largest spontaneous uptake of 2-DG was found in the central nucleus of the inferior colliculus (ICC). When compared to the control group, the ICC also showed the largest acoustically evoked response in long- (32\%) and short-term (16\%) groups among the various structures of the auditory system (Table II). However, the difference in ICC labeling between long- and short-term groups (14\%) was smaller than the one found in the LSO $(22 \%)$,
VCN (17\%) and TB (16\%). Therefore, although the ICC showed very high levels of metabolic activity, the effect of long-term habituation in the ICC was smaller or about the same found in more peripheral structures.

The external nucleus of the inferior colliculus (ICE), which surrounds the body of the colliculus, showed a considerably smaller level of 2-DG uptake (Fig. 4). Both the ICE and ICC showed acoustically evoked increases in the short-term rats as compared to the control group. However, only the ICC showed a significant difference between long- and short-term groups. So the ICE, which receives multisensory information, was not part of the auditory pathway involved in long-term habituation.

\section{Medial geniculate and auditory cortex}

The medial geniculate (MG) and auditory cortex (AC) showed no significant labeling differences

\section{LONG}
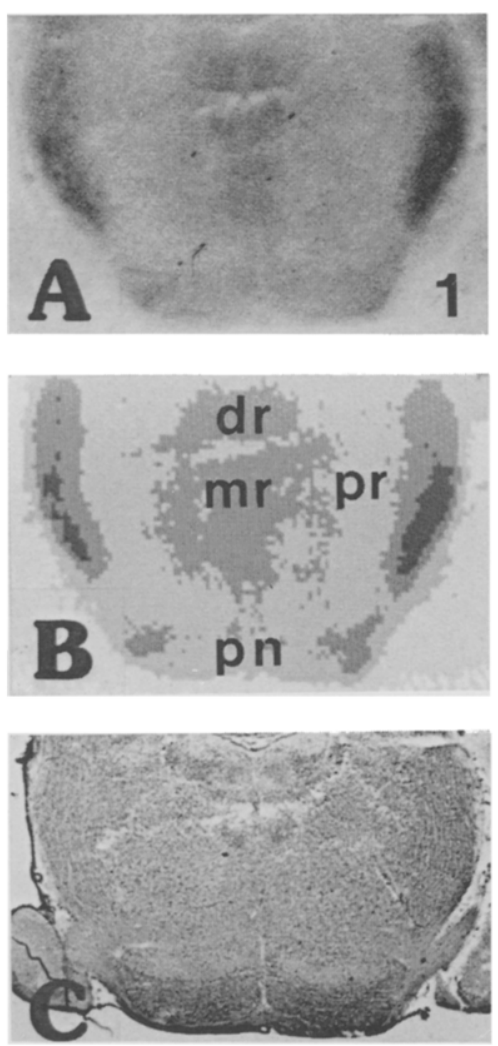

SHORT
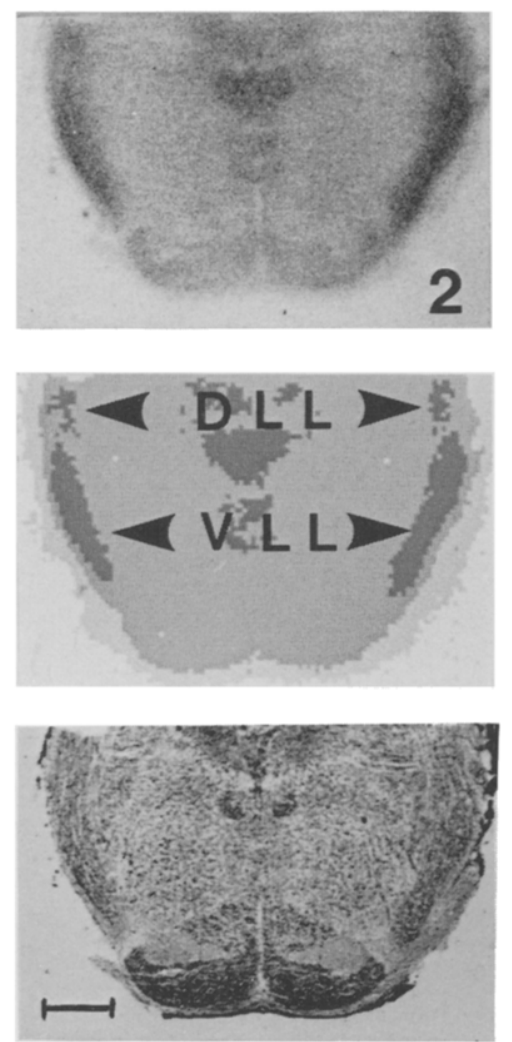

CONTROL
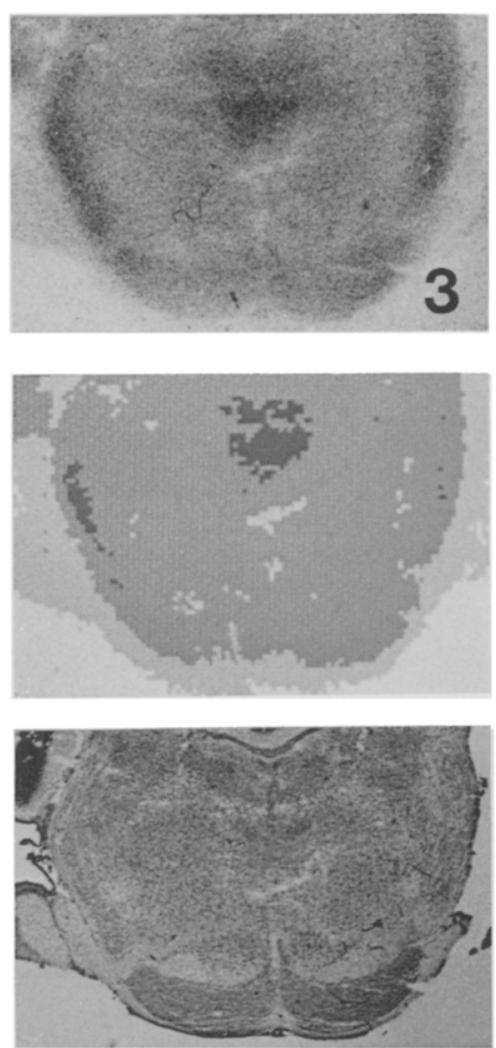

Fig. 3. Incorporation of 2-DG in the nuclei of the lateral lemniscus of rats from Groups $1-3$. The arrowheads of row $B$ point to DLL and VLL from dorsal to ventral, respectively. DLL, dorsal lateral lemniscus n.; VLL, ventral lateral lemniscus n.; dr, dorsal raphe $\mathrm{n}$.; mr, median raphe $\mathrm{n}$.; $\mathrm{pn}$, pontine $\mathrm{n}$.; $\mathrm{pr}$, pontine reticular formation. Bar $=1 \mathrm{~mm}$. 


\section{LONG}
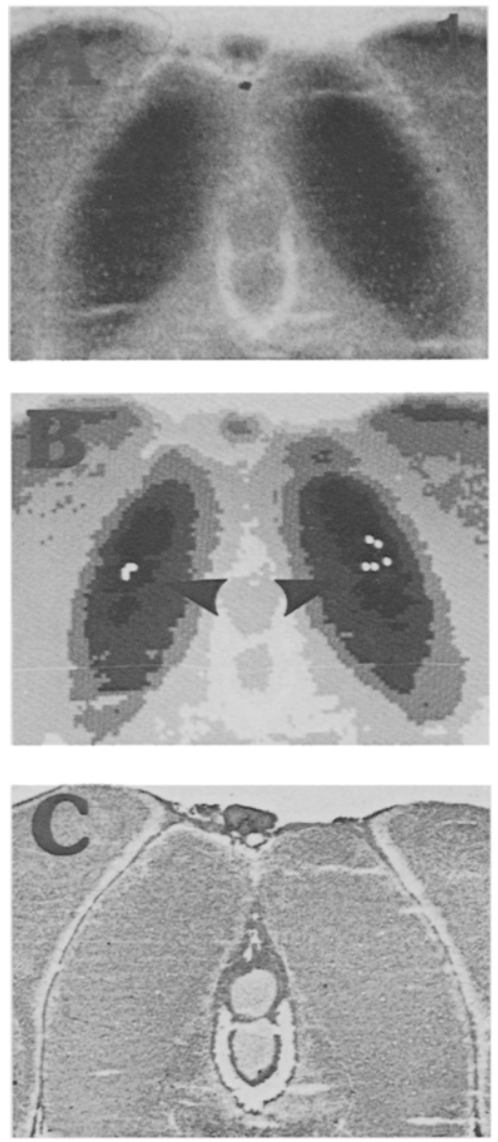

SHORT
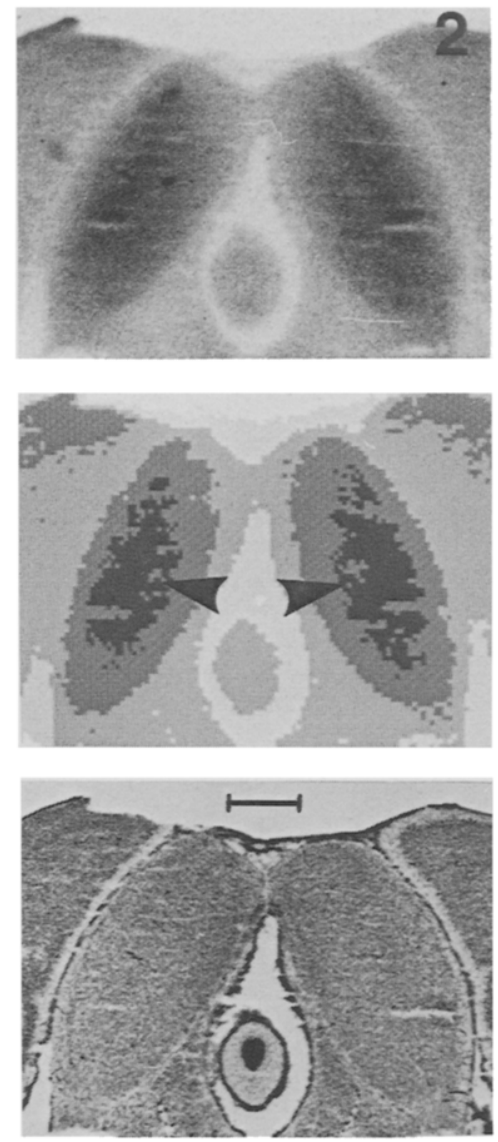

\section{CONTROL}
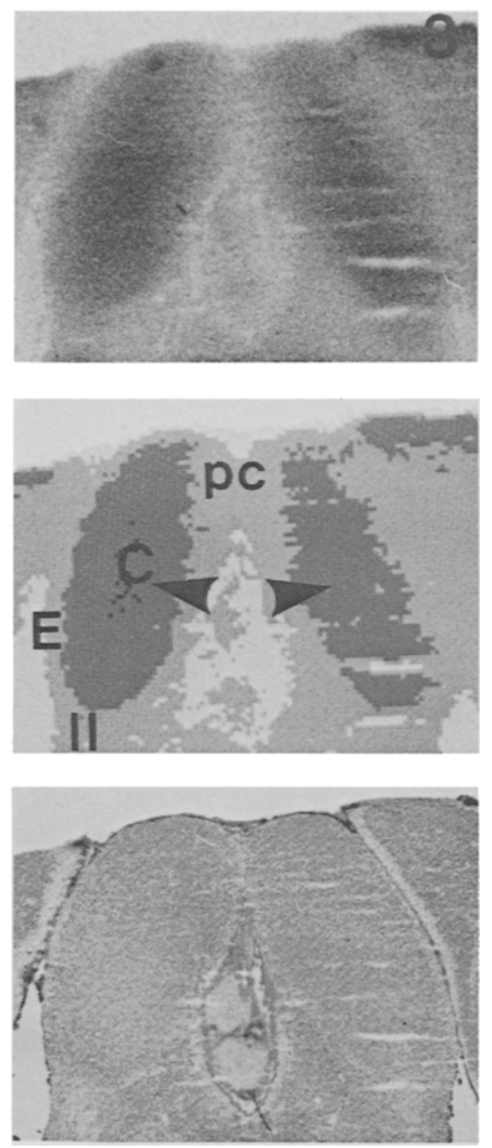

Fig. 4. Incorporation of 2-DG in the inferior colliculus of rats from Groups 1-3. The arrowheads in row B point to the region of maximal labeling (indicated by white dots in $B_{1}$ ). Note that the spontaneous labeling of the unstimulated rat (CONTROL) is high relative to other auditory structures (e.g., compare with cochlear nuclei in Fig. 1). Note the enhanced labeling in the central (C) but not in the external (E) nuclei of the LONG as compared to the SHORT columns. Other abbreviations: pc, paracentral cortex; ll, lateral lemniscus. Bar $=1 \mathrm{~mm}$.

between long- and short-term groups (Fig. 5). But in contrast to all the other auditory structures, the $\mathrm{MG}$ and $\mathrm{AC}$ showed mean differences between shortterm and control groups that were larger than the differences between long-term and control groups (Table II). Therefore, the enhancements in auditory patterns of 2-DG uptake dependent on long-term habituation appear to terminate at the level of the inferior colliculus.

\section{DISCUSSION}

The most important outcome of this study was the identification of a number of auditory structures with significant alterations in their metabolic response to an acoustic stimulus as a result of long-term habituation, a near universal form of behavioral plasticity considered one of the simplest forms of learning ${ }^{13,25}$. The results of this study provide the first demonstration of how a nonassociative learning experience such as long-term habituation modifies the metabolic activity of the auditory system. As in the case of our previous 2-DG studies of Pavlovian conditioning ${ }^{6,8}$, a 2-DG approach successfully visualized learningrelated changes in the auditory system of the intact rat. The purpose of this discussion is to elaborate on the specific metabolic alterations observed and their possible functional implications for long-term habituation of the acoustic startle response. 


\section{LONG}
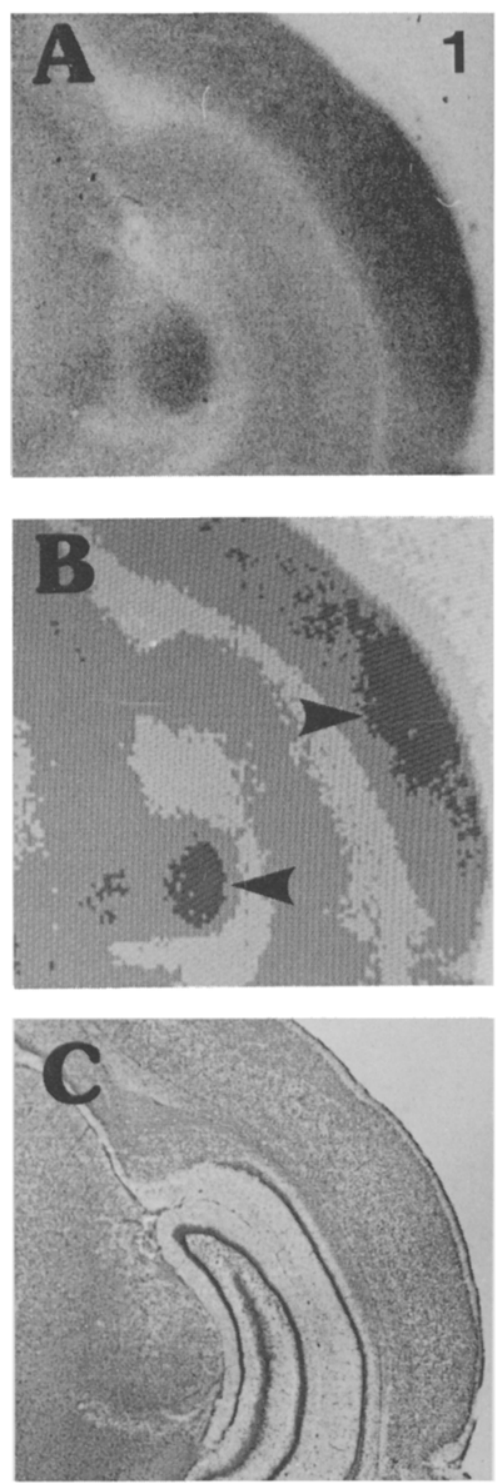

SHORT
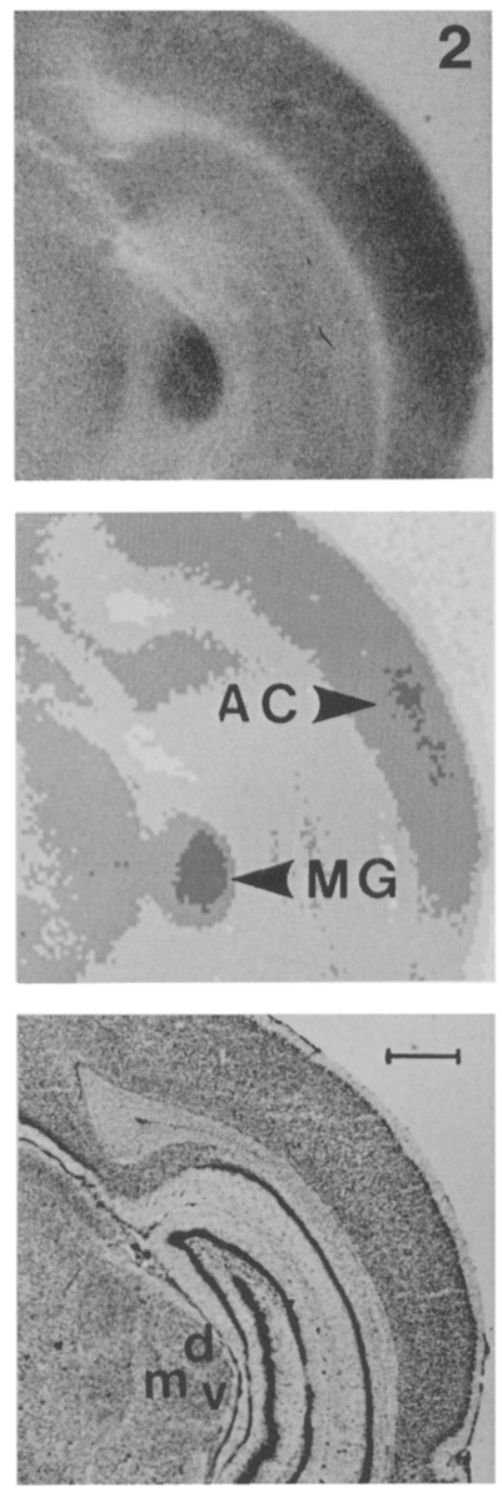

CONTROL
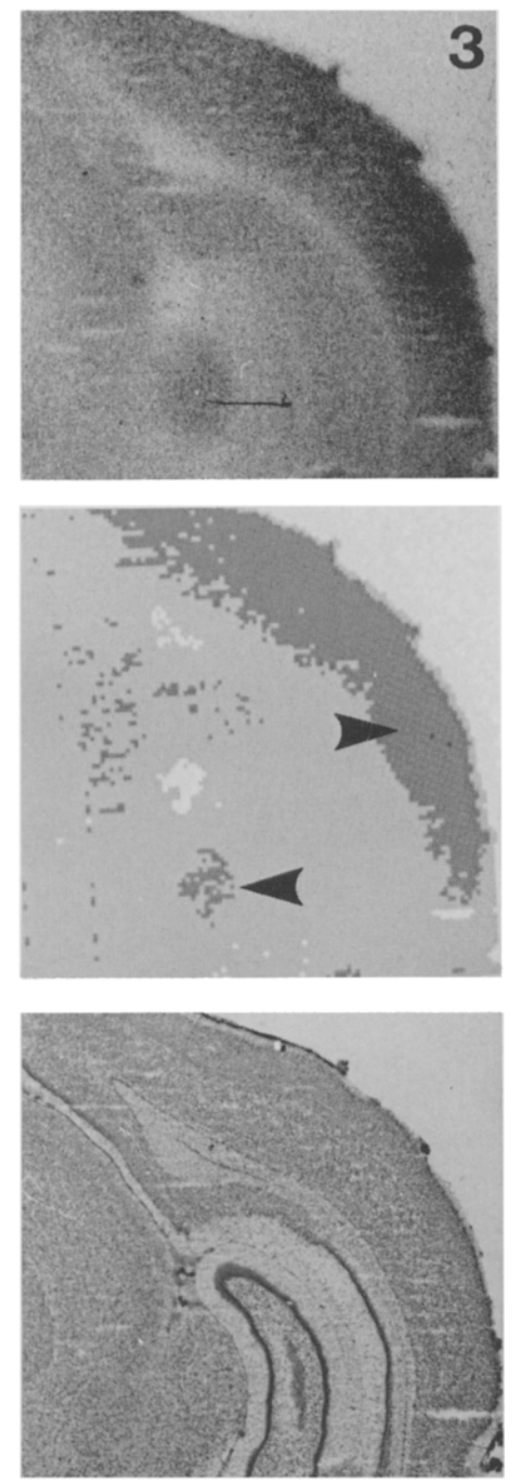

Fig. 5. Incorporation of 2-DG in the medial geniculate (MG) and the auditory cortex (AC) of rats from Groups 1-3. Row A autoradiographs show no clear differences in labeling between the MG and AC in LONG and SHORT rats. But these structures appear with lower intensity of labeling in the CONTROL rat. The dorsal (d) division of MG shows a slightly greater intensity of labeling in the SHORT as compared to the LONG rat. The other abbreviations stand for the medial ( $\mathrm{m}$ ) and ventral (v) divisions of $\mathrm{MG}$. Bar $=1 \mathrm{~mm}$.

\section{Central feedback control of peripheral auditory input}

Perhaps one of the most interesting specific findings of this study was that the largest difference in 2-DG uptake between long- and short-term habituated rats was localized in the lateral superior olivary nucleus (LSO). This is particularly relevant for the question of central control of peripheral auditory input during habituation, because LSO sends the largest number of descending efferents to the cochlea via the olivocochlear bundle ${ }^{27}$. The enhancement of LSO activity is suggestive of a central feedback mechanism that cannot be accounted for by some peripheral effect such as contraction of middle ear muscles ${ }^{25}$. The LSO 
metabolic activation suggests that long-term habituation to a repeated sound may involve a feedback mechanism relayed by the olivocochlear bundle whereby central auditory structures can exert influence on auditory input from the periphery. Such a mechanism may actively contribute to the decrement of acoustic startle responding to habituated sounds.

Early findings also support the idea that acoustically-evoked cochlear responses can be modified by central mechanisms during habituation ${ }^{13}$. Since the pioneer work of Hernandez-Peon ${ }^{13}$, a role for central control of peripheral auditory input has been actively debated ${ }^{13-15,21,22,25}$. For example, selective attention away from the auditory modality modifies active micromechanical properties of the cochlea via olivocochlear efferents ${ }^{22}$. It is tempting to speculate that both in long-term habituation and selective attention to nonauditory stimuli, there is a similar process of 'rejection' of auditory information (as indicated by the reduced behavioral responses evoked by the 'irrelevant' acoustic stimulus), and thus a similar central mechanism, via the olivocochlear bundle, may operate in both cases. But further work with methods suitable to test this hypothesis is needed.

\section{Learning mechanisms extrinsic to the primary acous-} tic startle circuit

A mechanism involving LSO feedback to the cochlea may work in conjunction with learningrelated processes taking place in brainstem auditory nuclei beyond the primary acoustic startle circuit ${ }^{2,3}$. The finding of enhanced metabolism from the cochlear nuclei to the inferior colliculus is compatible with the idea that active processes of neuronal plasticity were operating throughout the lower auditory system during long-term habituation ${ }^{13,15}$. After daily sessions of stimulation, peripheral auditory nuclei may become involved in a memory mechanism such as proposed by Sokolov ${ }^{24}$ in which new acoustic stimuli are compared with a representation of the habituated sound. A correct matching of the presented acoustic stimulus with the habituated stimulus, expressed as enhanced responses of lower auditory nuclei, may then trigger specific parallel outputs relevant for the modulation of the acoustic startle reflex during long-term habituation.

One of such outputs may be the LSO feedback to the cochlea via the olivocochlear bundle. This may be involved in the modification of the cochlear response to subsequent presentations of the habituated sound. A second output from auditory nuclei directed to the midbrain reticular formation may serve to modulate the arousing effects produced by the habituated sound ${ }^{6,13}$. In fact, the metabolic activity of the midbrain reticular formation is lower in long- as compared to short-term habituated animals ${ }^{4,10}$. A third output from the auditory nuclei to the cerebellar vermis may serve to influence the motor control of the startle response $e^{4,18}$. There are rich projections from the brainstem auditory nuclei to the vermis, in particular to some rostral regions of the vermis that show striking increases in 2-DG uptake during long- as compared to short-term habituation of the acoustic startle response ${ }^{10}$. Furthermore, lesions to the cerebellar vermis ${ }^{18}$ and the midbrain reticular formation ${ }^{16}$ have been shown to attenuate long-term habituation of the acoustic startle response in rats without affecting short-term habituation or altering initial response levels.

However, lesion studies of the effects of damage to auditory structures on long-term habituation are more difficult to assess because damage to any element in the auditory pathway reduces normal auditory responsiveness in addition to any interference with long-term habituation. Nevertheless, the available lesion data show no significant effects of damage to the auditory cortex or medial geniculate nucleus on long-term habituation ${ }^{16-18}$. This is in agreement with our data, which showed no differences in the metabolic activities of the auditory cortex and the medial geniculate nucleus between the long- and short-term groups. In the case of damage to the inferior colliculus, there have been reports showing conflicting results ${ }^{11,16,19}$, but the consensus is that deficits following inferior colliculus lesions involve the alteration of processes other than long-term habituation ${ }^{16}$. This type of conclusion from lesion studies of the inferior colliculus appears understandable in view of our 2-DG results which showed changes in the central (ICC) but not in the external nucleus (ICE) of the inferior colliculus.

Our data suggest that extrinsic control of the primary acoustic startle response circuit by auditory structures is mediated at the brainstem level. Reports of deficits in long-term habituation resulting 
from decerebration ${ }^{17}$ are more likely the result of interference with ascending midbrain reticular projections important for arousal modulation ${ }^{7}$ than the results of affecting auditory processes relevant for long-term habituation of the acoustic startle response.

Increase vs decrease of signal value in the auditory system

The present results are at variance with the effects of Pavlovian conditioning on the auditory system. Our previous 2-DG studies involving the signal conditioning of an acoustic stimulus paired with aversive reticular stimulation showed that the ICC was the auditory structure with the largest metabolic enhancement ${ }^{6}$. During classical conditioning with paired acoustic and aversive stimuli, the ICC showed an increase in 2-DG uptake that was $100 \%$ greater than the increase found in $\mathrm{LSO}^{6}$. Furthermore, the medial geniculate nucleus and the auditory cortex showed metabolic increases during classical conditioning ${ }^{8}$ but not during long-term habituation.

In the case of conditioning, an initially ineffective acoustic stimulus acquired a predictive signal value and effectively elicited a behavioral response after pairing with an aversive stimulus. This was mediated by activity increases at all levels of the auditory

\section{REFERENCES}

1 Cassella, J.V. and Davis, M., The design and calibration of a startle measurement system, Physiol. Behav., 36 (1986) 377-383.

2 Davis, M. and File, S.E., Intrinsic and extrinsic mechanisms of habituation and sensitization: implications for the design and analysis of experiments. In H.V.S. Peeke and L. Petrinovich (Eds.), Habituation, Sensitization, and Behavior, Academic Press, Orlando, FL, 1984, pp. 287-323.

3 Davis, M., Gendelman, D.S., Tischler, M.D. and Gendelman, P.M., A primary acoustic startle circuit: lesion and stimulation studies, J. Neurosci, 2 (1982) 791-805.

4 Finkenstädt, T. and Ewert, J.-P., Stimulus-specific longterm habituation of visually guided orienting behavior toward prey in toads: a ${ }^{14} \mathrm{C}-2 \mathrm{DG}$ study, J. Comp. Physiol., 163 (1988) 1-11.

5 Gonzalez-Lima, F., Midbrain reticular stimulation produces patterns of metabolic activation and suppression in the cerebellum and vestibular nuclei: a 2-deoxyglucose study, Brain Research, 412 (1987) 275-284.

6 Gonzalez-Lima, F. and Scheich, H., Neural substrates for tone-conditioned bradycardia demonstrated with 2-deoxyglucose. I. Activation of auditory nuclei, Behav. Brain Res., 14 (1984) 213-233.

7 Gonzalez-Lima, F. and Scheich, H., Ascending reticular pathway, in particular at the $\mathrm{ICC}^{6,8}$. By contrast, in the case of long-term habituation, the response to the acoustic stimulus was effectively reduced after the animal learned not to respond to a repeated stimulus whose consequences were neither aversive nor rewarding. This was mediated by activity increases at lower levels of the auditory pathway, particularly at the LSO. The auditory system clearly plays different roles in these somewhat opposite forms of auditory learning involving an increase (conditioning) or a decrease (habituation) of the signal value of an acoustic stimulus. The findings support previous 2-DG and electrophysiological studies of learning ${ }^{4-14,20,21}$ which show that auditory responses of behaving animals to acoustic stimuli are dependent not only on the physical parameters of a stimulus, but also on its learned behavioral significance.

\section{ACKNOWLEDGEMENTS}

Thanks are due to E. Gonzalez-Lima and C. Rivera-Quiñones for their assistance. This work was supported in part by grants from NATO (860813), DFG (Fi 315/3), NIH (RR05814) and Texas A\&M University.

activating system in the rat: a 2-deoxyglucose study, Brain Research, 344 (1985) 70-88.

8 Gonzalez-Lima, F. and Scheich, H., Neural substrates for tone-conditioned bradycardia demonstrated with 2-deoxyglucose. II. Auditory cortex plasticity, Behav. Brain Res., 20 (1986) 281-293.

9 Gonzalez-Lima, F. and Scheich, H., Classical conditioning of tone-signaled bradycardia modifies 2-deoxyglucose uptake patterns in cortex, thalamus, habenula, caudateputamen and hippocampal formation, Brain Research, 363 (1986) 239-256.

10 Gonzalez-Lima, F., Finkenstädt, T. and Ewert, J.-P, Neural substrates for long-term habituation of the acoustic startle reflex in rats: a 2-deoxyglucose study, Neurosci. Lett., 96 (1989) 151-156.

11 Groves, P.M., Wilson, C.J. and Boyle, R.D., Brainstem pathways, cortical modulation, and habituation of the acoustic startle response, Behav. Biol., 10 (1971) 391-418.

12 Harvey, J.A., Winsky, L., Schindler, C.W., McMaster, S.E. and Welsh, J.P., Asymmetric uptake of 2-deoxy$\mathrm{D}-\left[{ }^{14} \mathrm{C}\right]$ glucose in the dorsal cochlear nucleus during Pavlovian conditioning in the rabbit, Brain Research, 449 (1988) 213-224.

13 Hernandez-Peon, R., Neurophysiological correlates of habituation and other manifestations of plastic inhibition, EEG Clin. Neurophysiol., Suppl. 13 (1960) 101-114. 
14 Hillyard, S.A. and Kutas, M., Electrophysiology of cognitive processing, Annu. Rev. Psychol., 34 (1983) 33-61.

15 Horn, G., Neuronal mechanisms of habituation, Nature, 215 (1967) 707-711.

16 Jordan, W.P. and Leaton, R.N., Habituation of the acoustic startle response in rats after lesions in the mesencephalic reticular formation or in the inferior colliculus, Behav. Neurosci., 97 (1983) 710-724.

17 Leaton, R.N., Cassella, J.V. and Borszcz, G.S., Shortterm and long-term habituation of the acoustic startle response in chronic decerebrate rats, Behav. Neurosci., 99 (1985) 901-912.

18 Leaton, R.N. and Supple, Jr., W.F., Cerebellar vermis: essential for long-term habituation of the acoustic startle response, Science, 232 (1986) 513-515.

19 Leitner, D.S. and Cohen, M.E., Role of the inferior colliculus in the inhibition of acoustic startle in the rat, Physiol. Behav., 34 (1985) 65-70.

20 Miller, J.M., Pfingst, B.E. and Ryan, A.F., Behavioral modification of response characteristics of cells in the auditory system. In C.D. Woody (Ed.), Conditioning: Representation of Involved Neural Functions, Plenum, New York, 1982, pp. 345-362.
21 Picton, T.W. and Hillyard, S.A., Human auditory evoked potentials. II. Effects of attention, EEG Clin. Neurophysiol., 36 (1974) 191-199.

22 Puel, J.-L., Bonfils, P. and Pujol, R., Selective attention modifies the active micromechanical properties of the cochlea, Brain Research, 447 (1988) 380-383.

23 Sharp, F.R., Kilduff, T.S., Bzorgchami, S., Heller, H.C. and Ryan, A.F., The relationship of local cerebral glucose utilization to optical density ratios, Brain Research, 263 (1983) 97-103.

24 Sokolov, E.N., The neuronal mechanisms of the orienting reflex. In E.N. Sokolov and O.S. Vinogradova (Eds.), Neuronal Mechanisms of the Orienting Reflex, Lawrence Erlbaum Associates, New Jersey, 1975, pp. 217-235.

25 Thompson, R.F. and Spencer, W.A., Habituation: a model phenomenon for the study of neuronal substrates of behavior, Psychol. Rev., 73 (1966) 16-43.

26 Toga, A.W. and Collins, R.C., Glucose metabolism increases in visual pathways following habituation, Physiol. Behav., 27 (1981) 825-834.

27 Warr, W.B. and Guinan, Jr., J.J., Efferent innervation of the organ of Corti: two separate systems, Brain Research, 173 (1979) 152-155. 